\title{
Frank M. Gasparini-A Tribute to an Outstanding Scientist and Educator, in Celebration of His 70th Birthday
}

\author{
Eckhard Krotscheck • Hong Luo
}

Published online: 22 September 2012

(C) Springer Science+Business Media New York 2012

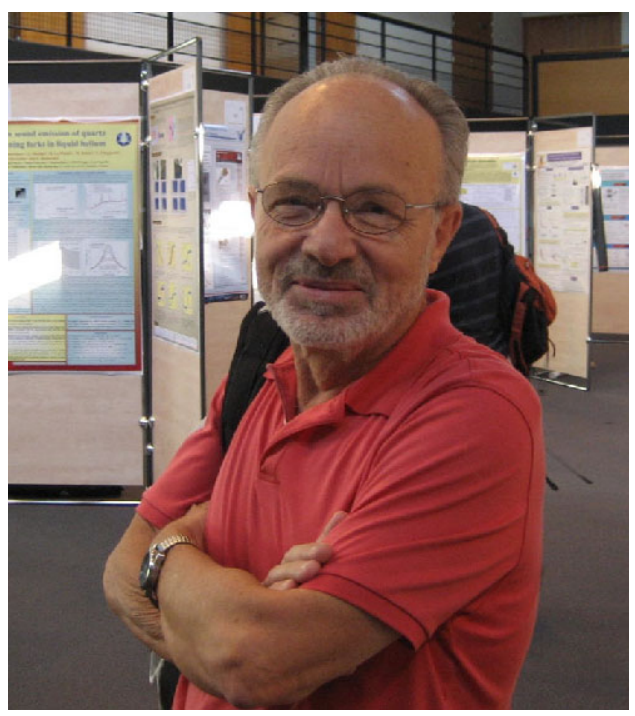

(Photo courtesy of Horst Meyer)

Frank joined the Physics Department at the University at Buffalo (UB) in 1973 as an Assistant Professor, was promoted to the rank of Associate Professor in 1978 and Full Professor in 1984. He became a UB Distinguished Professor in 2007, a fitting

This introduction was written with the help of Mark O. Kimball and Justin K. Perron.

E. Krotscheck $(\bowtie) \cdot$ H. Luo

Department of Physics, University of Buffalo SUNY, Buffalo, NY 14260, USA

e-mail: eckhardk@buffalo.edu 
recognition of his exemplary scientific contributions and outstanding service to the University. In 2012, Frank became the endowed Rustgi Professor for his contributions to his research field and education.

A central theme to Frank's research at UB has been the methodical study of liquid helium confined to be less than three-dimensional near the superfluid transition. Early work, reviewed in the book chapter titled "Finite Size Scaling of Confined Helium Near $T_{C}$," with I. Rhee, found in Progress in Low Temperature Physics, Vol. 13, D.F. Brewer ed. (North Holland, 1992) pp. 1-90, focuses on helium confined in porous glasses and packed powder. This helped lay the foundation for the study of finite-size scaling (FSS) as it pertains to helium. Inhomogeneity in these early confinements, which blurs finite-size effects, was overcome by developing a new confinement technique. Cells made from silicon wafers patterned using standard nanofabrication technology and made superfluid tight via direct wafer bonding produced uniform confinements. This allowed for a systematic investigation of thermodynamic properties near the superfluid transition in 2,1 , and 0 dimensions by confining helium to slabs, channels, and boxes, respectively. Probing the specific heat and superfluid density of helium in these precise geometries allowed one to experimentally observe the crossover of bulk-like helium to all three lower spatial dimensions. This elegant work elucidated the many successes of FSS and also made apparent some areas where FSS is failing. The review article titled "Finite-size scaling of ${ }^{4} \mathrm{He}$ at the superfluid transition" with Mark O. Kimball, Kevin P. Mooney, and Manuel Diaz-Avila, in Reviews of Modern Physics, 80, 1009-1059, 2008, summarizes this important contribution. Frank's current research is focused on a proximity effect in helium that was discovered during studies where helium is confined to small homogeneous boxes linked with shallow fill channels. A report of this is found in Coupling and Proximity effects in the superfluid transition in ${ }^{4} \mathrm{He}$ dots with Justin K. Perron, Mark O. Kimball and Kevin P. Mooney Nature Phys. 6, 499-502 (2010). The existence of this effect casts light on some of the FSS results and also poses very interesting questions regarding proximity effects and the correlation length of helium.

Frank's contribution to physics goes beyond his research. To the university community at UB, Frank has been an outstanding educator, a colleague, and a leader in the Department, serving in various capacities, including the Associate Chair and the Chair of the Department for a total of 14 years. The Department experienced unprecedented growth during his tenure as the Chair. Under his stewardship, both the quality and culture of the Department have seen marked improvement. Frank has received numerous awards and recognitions, including becoming a Fellow of the American Physical Society in 1990, receiving the SUNY Chancellor Award for Excellence in Teaching in 1996 and the Provost's Sustained Achievement Award in 2002.

Frank is known for his dedication to education. With all his research and administrative responsibilities over the years, he has been first and foremost an educator. Regardless of his workload at any given time, his time for preparation for his classes and for graduate students remained the same. This sense of responsibility and discipline also goes into his instruction and his requirement for his students. His classes are challenging and rewarding at the same time. Frank is the lead author of 4 volumes of laboratory manuals for four introductory physics courses, with proceeds going back to instruction, which have been used in the Department for over 20 years and by tens 
of thousands of students. After stepping down as Chair, he has been working with the manuals' co-author, Prof. Petrou, and the laboratory instructor to update the manuals so that they keep pace with changing technologies.

The Physics Department at UB made great strides in many key areas under his leadership. For example, the number of publications from the Department was tripled during this period, and so was the annual number of student co-authors. The Department presently has 8 National Science Foundation CAREER winners, more than the counterparts in elite institutions. Half of the awards were received during Frank's tenure as Chair. His contributions to these extraordinary accomplishments can be easily seen from his hands-on mentoring of our junior faculty members. Frank continued to be a great source of wisdom and advice after stepping down as Department Chair.

This special issue with articles from colleagues and friends is dedicated to him as a scientist, educator, and friend. 